\title{
Effect of Adjusting the Challenge-Skill Balance for Occupational Therapy in a Recovery Rehabilitation Unit: A Pilot Study
}

\author{
Ippei Yoshida ${ }^{1,2}$, Kazuki Hirao ${ }^{3}$, Ryuji Kobayashi ${ }^{1}$ \\ ${ }^{1}$ Department of Occupational Therapy, Graduate School of Human Health Sciences, Tokyo Metropolitan University \\ ${ }^{2}$ Department of Rehabilitation, Harue Hospital \\ ${ }^{3}$ Department of Occupational Therapy, Kibi International University Graduate School of Health Sciences
}

\begin{abstract}
The primary goal of occupational therapy is to enable people to participate in normal everyday activities. To understand and support a client's desired activities, it is necessary to share the meaning of occupation between the therapist and client. One concept that has been used to capture the psychological state for an activity is flow. Therefore, we devised a process of adjusting the challenge-skill balance for occupational therapy based on flow theory.

In this pilot study, we aimed to verify whether adjusting the challenge-skill balance for occupational therapy improved subjective quality of life in a recovery rehabilitation unit. To inform the future design of randomized controlled trials, we therefore compared standard occupational therapy with this new approach. Both interventions took place from recovery rehabilitation unit entry to discharge, and outcomes (continuous variables) were analyzed using a Bayesian approach explored with generalized linear mixed modeling.

Among a total of 22 patients, those receiving the new approach showed a significant improvement in Ikigai-9 (quality of life) compared with those receiving standard occupational therapy. The mean improvement was $4.44 \pm 2.17$ with a $95 \%$ credible interval of $0.104-8.713$. The dependence factor, effective sample size, and autocorrelation time were 3.769 , 8093 , and 6.18, respectively. We conclude that adjusting the challenge-skill balance during occupational therapy could improve a client's subjective quality of life.
\end{abstract}

Keywords: occupational therapy process, flow model, quality of life, recovery rehabilitation unit

(Asian J Occup Ther 15: 11-18, 2019)

\section{Introduction}

Occupational therapy is a client-centered profession concerned with promoting health and well-being through occupation. The primary goal of occupational therapy is to enable people to participate in normal everyday activities [1]. In occupational therapy, appropriate activities are typically set based on a comprehensive assessment of a client's physical and mental functions, occupational performance, and personal factors, as well as any relevant environmental factors. Several practical frameworks exist, including the Occupational Therapy

Received: 17 February 2018, Accepted: 26 December 2018 Corresponding to: Ippei Yoshida, Department of Occupational Therapy, Graduate School of Human Health Sciences, Tokyo Metropolitan University, 7-2-10, Higashiogu, Arakawa-ku, Tokyo, 116-8551, Japan e-mail: ippe.i.yoshi@gmail.com

(C2019 Japanese Association of Occupational Therapists
Practice Framework [2], the Occupational Therapy Intervention Process Model [3], and the Management Tool for Daily Life Performance [4], together with important theories, such as the Canadian Model of Occupational Performance [5] and Model of human occupation [6]. However, despite attempts to ensure that occupational therapists provide client-centered therapy, it has been reported that there can be differences in the perception of skill and challenge difficulty between the occupational therapist and client [7].

To understand and support a client's desired activities, it is necessary to share the meaning of occupation between the therapist and client. In other words, subjective assessments about a client's occupation should be acquired in a way that facilitates easy recognition. One concept that has been used to capture the psychological state for an activity is "flow" [8]. Csikszentmihalyi reported that flow occurs when individuals are "so intensely involved in an activity that nothing else seems to 
matter; the experience itself is so enjoyable that people will do it even at great cost, for the sheer sake of doing it" [9]. Flow can contribute to increased happiness, self-esteem, work productivity, and joy of life [10-13]. Additionally, studies have described the relationship between the experience of flow and everyday life activities $[6,14-16]$.

According to flow theory, flow can also be defined operationally according to a challenge-skill balance called the "flow model" [8]. This model proposes that flow occurs when the perceived level of challenge provided by the activity is in balance with the participant's level of skill. By contrast, activities in which the individual has high perceived skill relative to the challenge might lead to boredom, whereas activities in which there is low perceived skill and high perceived challenge might lead to anxiety. As research based on the flow model, there were analyzes targeting activities engaged by students and occupational therapists. [17-19], while other analyses have looked at the relationship between flow and health-related quality of life (QoL) in elderly people [20-22]. We previously analyzed the activities supported by occupational therapy among elderly people in an adult day program and showed a difference in recognition about the activity, consistent with previous research $[7,22]$. Based on the flow model, we thought that adjusting the challenge-skill balance might facilitate occupational therapy. In a randomized controlled trial, we examined the effect of occupational therapy in which the challenge-skill balance was adjusted for elderly people who used an adult day program, and we showed improvements in health-related QoL [23].

However, since this previous study targeted only one specific activity in the maintenance rehabilitation phase, the influence of the intervention period and the effectiveness of support for multiple activities have not been clarified. Therefore, in this study, we conducted the process adjusting the challenge-skill balance (called adjusting the challenge-skill balance for occupational therapy; ACS-OT) in a situation that supports multiple activities at recovery phase, we examined whether ACS-OT was more universally effective in occupational therapy. Our aim was to verify if ACS-OT improved subjective QoL in a recovery rehabilitation unit.

\section{Method/Design}

\section{Method}

This controlled clinical trial was designed to compare the ACS-OT method (experimental group) with standard occupational therapy (control group). The primary outcome was change in subjective QoL (ikigai) for both groups from admission (pre) to discharge (post).
The secondary outcomes were changes in health-related QoL, flow experience, and ADLs. The study protocol was approved by the Ethics Review Committee of the Harue hospital (No.29-1).

\section{Inclusion and exclusion criteria}

From May 2017 to November 2017, Clients were included if they were admitted to the recovery rehabilitation unit of Harue hospital with either cerebrovascular disease or musculoskeletal disease. On admission to the recovery rehabilitation unit, we excluded clients aged $<50$ years and $\geq 100$ years, as well as those whose Mini Mental State Examination scores were $\leq 23$ points [24].

\section{Procedure}

\section{Intervention}

Occupational therapy was provided according to the guidelines of the American Occupational Therapy Association [2] in both the experimental and control groups. All interventions were implemented by occupational therapists experienced ( $\geq 200$ hours) in delivering client-centered occupational therapy. In reference to the Graff's research design [25], the criteria for the amount of experience was set to more than 6 cases (40 minutes, 50 days, 6 cases, total 200 hours). Moreover, all had received at least 50 hours of training for ACS-OT (30 minutes, 50 days, 2 cases, Total 50 hours). Occupational therapy was conducted individually and was focused on the performance of activities. The key difference between groups was whether the evaluation and intervention were conducted based on an assessment of the challenge-skill balance. Treatment consisted of 40-60 minutes per day, six times in total in a week. The study period was from admission to discharge from the recovery rehabilitation unit.

\section{Experimental group}

The ACS-OT process was implemented in the following way. In the first session, the therapist assessed the client's problems in daily living using the Canadian Occupational Performance Measure [26]. Based on the problems identified, some activities that could be supported by occupational therapy were selected. In the second session, the client performed the tasks related to selected activities and evaluated the "challenge level" and "skill level" as follows: challenge level defined as the "challenge of the activity" and rated on a seven-point scale from "very simple" (1) to "very difficult" (7); and "skill level" was defined as "your skills in the activity," rated from "not at all skillful" (1) to "very skillful" (7) $[18,27]$. The therapist checked with the client why certain ratings were given. 
Subsequently, evaluation by the client and therapist in collaboration helped decide on the factors that made occupational performance difficult (i.e., challenge components, such as environment, execution time, and and size of the location in which the activity is carried out) and that improved performance (i.e., skill components, such as frequency, range, distance, accuracy, and dexterity related to activity). The challenge-skill balance for tasks was then changed based on these conclusions. The criteria for adjusting the challenge-skill balance was that the difference in evaluation of the challenge and skill levels between the therapist and client had to be one or less.

After the client performed the adjusted tasks, the challenge and skill levels were re-evaluated. If the challenge and skill levels were balanced, therapy was started to improve performance in those activities. If the challenge and skill levels were not balanced, the tasks were further adjusted and therapy was only started after they were balanced. This process was repeated at least once a week. It should be noted that, activity was changed (added or finished) according to the status of treatment progress. For example, in the initial session, activities such as walking and toilets are targeted. Then, the toilet is achieved (finished), walking tasks are shifted to stairs and applied walking, bathing and cooking are added.

\section{Control group}

The first and second sessions were conducted similarly to the experimental group, with the exception that therapists were not allowed to confirm the client's subjective perceptions of the challenge posed by, or skill needed to complete, the activities. From the third session, therapists assessed the client's performance and conducted therapy in a typical manner, following the general guidelines for occupational therapy [2].

\section{Outcomes}

\section{Subjective $Q o L$}

We measured subjective QoL using the Ikigai9 [28], a client's psychological measure of feeling a reason for living (ikigai). It consisted of nine items rated on 5-point scales (total score, 9-45 points).

\section{Health-related QoL}

Health-related QoL was assessed by the EuroQol-5 Dimensions (EQ-5D) [29]. The EQ-5D defines health in five dimensions (i.e., mobility, self-care, usual activities, pain and discomfort, and anxiety or depression). A visual analog scale (EQ-VAS) enables self-assessment on a scale from 0 (worst possible health) to 100 (best possible health) per dimension.

\section{Flow experience}

Flow experience was assessed using the Flow State Scale for Occupational Tasks [30] developed for clinical situations. This consists of 14 items and 3 factors (scores range from 7 to 98 ), and its reliability and validity have been confirmed [30].

\section{$A D L$}

ADLs were measured by the Functional Independence Measure (FIM) [31]. This comprises 18 items on 7-point scales that uniformly assess the severity of a client's disability and functional outcome from medical rehabilitation (overall score, 7 to 126).

\section{Organization}

The primary investigator plans and executes this research and supervises three research assistants. The research assistants obtained informed consent and performed procedures, measurements. Data entry and control were conducted by the primary investigator. A statistician also helped with the data analysis.

\section{Statistical analysis}

All statistical analyses were performed using EZR [32] and SAS $^{\circledR}$ (SAS Institute, Sally, NC, USA). Data were entered in Microsoft Excel 2016 and subsequently exported into EZR and SAS for analysis. Baseline characteristics were compared by chi-square tests for categorical variables and by independent $t$-tests and MannWhitney $U$ tests for continuous variables. The comparison of categories of activities targeted in two groups was analyzed using Fisher's exact test.

Each continuous outcome variable was analyzed using a generalized linear mixed model (GLMM). Considering the sample size of this study, outcome analysis adopted a Bayesian method that enables reasonable estimation even when the sample size is small. We took a Bayesian approach and explored the Markov chain Monte Carlo (MCMC) [33] fitting of GLMM, including the following as fixed effects: group allocation (experimental and control groups), time (pre and post), and the interaction of group and time. Participants were included as a random effect. MCMC is a method of generating a sample having a distribution characteristic matching the posterior distribution by Markov chain using Bayesian method and using it to calculate an estimate value of the objective variable. The convergence criterion for data in the MCMC fitting was set to a dependence factor of 5 for Raftery-Lewis Diagnostics [34]. In addition, autocorrelation time (ACT) and effective sample size (ESS) are also effective indices for 
the diagnosis of MCMC algorithm. The ACT measures dependency among Markov chain samples. The ESS estimates how many truly independent samples of a given parameter the output of the MCMC represents. High ESS or low ACT indicates good mixing of the Markov chain. Therefore, we also confirmed the ACT and ESS for MCMC convergence. All credible intervals were given with a $95 \%$ credible interval.

Since the Bayesian method only calculates the estimated value, effect sizes indicating the difference between the two groups with respect to each outcome after the intervention were calculated to show the magnitude of the effect in the experimental group.

\section{Results}

We included 22 clients (experiment Group; 11, control Group;11) and confirmed that the pre-test characteristics were well matched between the two study groups (Table 1). The total number of activities targeted by both groups was 81 (average per client: 3.68), ADL was 54 (66.6\%), and instrumental ADL was 20 (24.7\%). There was no significant difference in activity between the two groups (Fig. 1).

The changes from the pre-test to the post-test characteristics are shown in Table 2. Regarding each outcome after intervention, Ikigai-9 was $33.18 \pm 7.04$ in the experimental group and $26.64 \pm 8.98$ in the control group. EQ-5D was $0.668 \pm 0.091$ in the experimental group and $0.653 \pm 0.122$ in the control group, Flow state scale for occupational task was $82.27 \pm 8.90$ in the experimental group and $73.27 \pm 16.85$ in the control group. FIM total score was $118.73 \pm 4.29$ in the experimental group and $117.64 \pm 8.89$ in the control group. The results analyzed by MCMC for GLMM are shown in Table 3. The mean improvement in the Ikigai-9 between the experimental and control groups was $4.44 \pm 2.17$, with a $95 \%$ credible interval of $0.10-8.71$. The difference between the two groups in EQ-5D was $0.061 \pm$ 0.089 with a $95 \%$ credible interval of $-0.115-0.236$, in Flow state scale for occupational task was $-0.73 \pm 5.01$ with a $95 \%$ credible interval of $-10.88-8.94$, in FIM was $3.26 \pm 4.88$ with a $95 \%$ credible interval of $-6.37-$ 12.72. Thus, a significant difference was confirmed in Ikigai-9. Confirming the information on the convergence situation of sampling about MCMC analysis for Ikigai-9, The dependence factor, ACT, and ESS were 3.769, 6.178 , and 8093.4, respectively. In addition to the results of dependence factor (less than 5), ESS and ACT, posterior distribution by sampling data was in a smooth normal distribution state, indicating that appropriate sampling was being performed (Fig. 2).

There were no significant differences in the EQ-5D, Flow State Scale for Occupational Tasks or FIM between the experimental and control groups. Regarding the FIM, there was a significant difference between the

Table 1. Baseline data.

The results of the baseline data in the experimental and control groups were shown. The pre-test characteristics were well matched between the two groups.

\begin{tabular}{lccc}
\hline & Experimental Group (n=11) & Control Group (n=11) & $p$ value \\
\hline Age (M, SD) & $75.36(10.51)$ & $73.64(13.00)$ & $p=0.742$ \\
\hline Sex (n) & 3 & 6 & $p=0.387$ \\
Male & 8 & 5 & \\
Female & 3 & 5 & $p=0.659$ \\
\hline Disease (n) & 8 & 6 & $p=0.742$ \\
$\quad$ Cerebrovascular & $46.27(18.81)$ & $44.64(28.41)$ & $p=0.629$ \\
Musculoskeletal & $25.64(2.16)$ & $26.18(2.99)$ & $p=0.745$ \\
\hline Treatment period: Day (M, SD) & $60.45(18.64)$ & $63.36(22.59)$ & $p=0.212$ \\
\hline MMSE (M, SD) & $79.73(14.11)$ & $70.00(20.63)$ & $p=0.442$ \\
\hline EQ VAS (M, SD) & $31.09(10.15)$ & $28.00(8.25)$ & \\
\hline Flow state scale for Occupational task (M, SD) & & & \\
\hline Ikigai-9 (M, SD) & $63.36(14.35)$ & $65.36(8.66)$ & $p=0.696$ \\
\hline FIM (M, SD) & $33.09(2.12)$ & $33.27(3.90)$ & $p=0.237$ \\
Motor & $96.45(14.69)$ & $98.64(10.55)$ & $p=0.693$ \\
Cognitive & & & \\
Total &
\end{tabular}

Note. $\mathrm{M}=$ mean; $\mathrm{SD}=$ standard deviation; EQ-VAS = EuroQol-5 Dimension visual analog scale; MMSE $=$ Mini Mental State Examination; FIM $=$ Functional Independence Measure. 


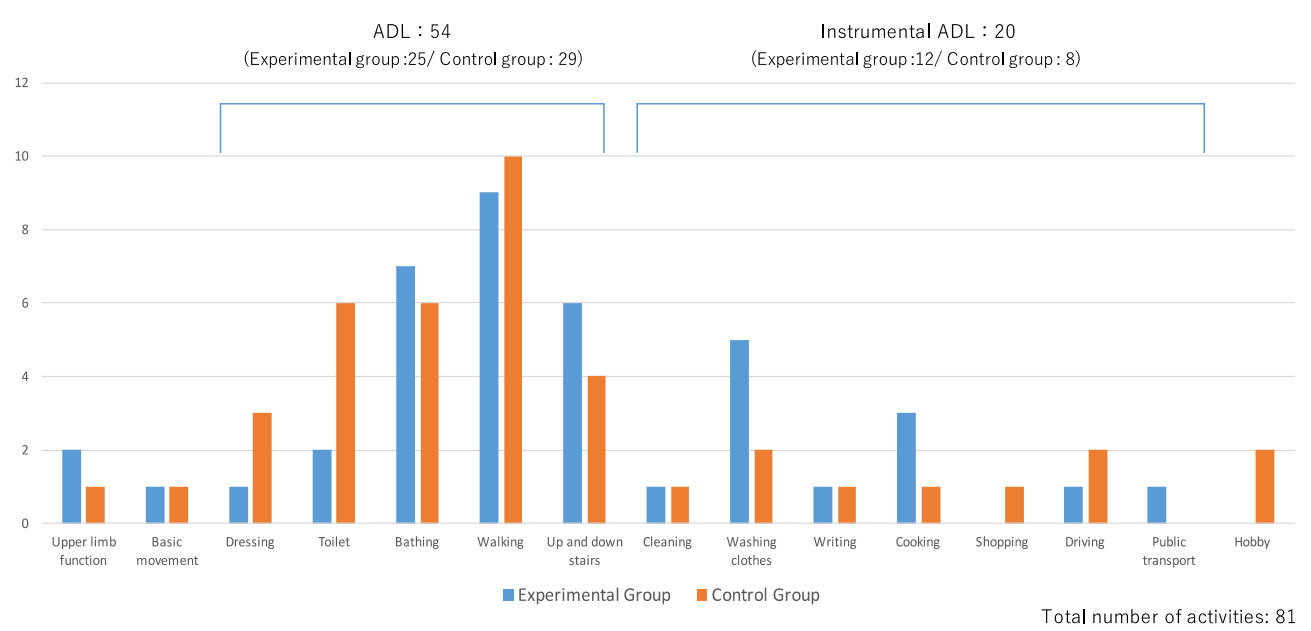

Fig. 1. Activities targeted by experiment group and control group.

In this figure, it showed the classification of the activities that was the target in the experiment group and the control group. As a result of Fisher's exact test, there was no significant difference in activity between the two groups.

Table 2. Pre and post data in experimental and control group.

Changes from pretest to post-test characteristics about Ikigai-9, EQ-5D/EQ VAS, Flow state scale for occupational task, and FIM between the experimental and control groups were shown. Effect sizes between two groups with respect to each outcome after the intervention were also shown.

\begin{tabular}{|c|c|c|c|c|c|}
\hline & \multicolumn{2}{|c|}{ Observed M (SD) } & \multicolumn{2}{|c|}{ Observed M (SD) } & Effect size $[95 \% \mathrm{CI}]$ \\
\hline & \multicolumn{2}{|c|}{ Experimental group $(\mathrm{n}=11)$} & \multicolumn{2}{|c|}{ Control group $(n=11)$} & \multirow{2}{*}{$\begin{array}{l}\text { Between two groups } \\
\text { after the intervention }\end{array}$} \\
\hline & pre & post & pre & post & \\
\hline Ikigai-9 & $31.09(10.15)$ & $33.18(7.04)$ & $28.00(8.25)$ & $26.64(8.98)$ & $0.78(-0.09$ to 1.65$)$ \\
\hline EQ-5D & $0.497(0.187)$ & $0.668(0.091)$ & $0.544(0.243)$ & $0.653(0.122)$ & $0.19(-0.70$ to 0.97$)$ \\
\hline EQ VAS & $60.45(18.64)$ & $77.09(15.43)$ & $63.36(22.59)$ & $72.27(20.42)$ & \\
\hline Flow state scale for occupational task & $79.73(14.11)$ & $82.27(8.90)$ & $70.00(20.63)$ & $73.27(16.85)$ & $0.67(-0.21$ to 1.50$)$ \\
\hline \multicolumn{6}{|l|}{ FIM } \\
\hline Total-score & 96.45 (14.69) & $118.73(4.29)$ & $98.64(10.55)$ & 117.64 ( 8.89) & $0.16(-0.69$ to 0.99$)$ \\
\hline Motor-score & $63.36(14.35)$ & $85.27(3.82)$ & $65.36(8.66)$ & $84.73(5.76)$ & \\
\hline Cognitive-score & $33.09(2.12)$ & $33.45(1.81)$ & $33.27(3.90)$ & $32.9(5.07)$ & \\
\hline
\end{tabular}

Note. $\mathrm{M}=$ mean; $\mathrm{SD}=$ standard deviation; $\mathrm{CI}=$ confidence interval; $\mathrm{EQ}-5 \mathrm{D}=$ EuroQol-5 Dimension;

EQ-VAS = EuroQol-5 Dimension visual analog scale; FIM = Functional Independence Measure;

Effect sizes between two groups $=$ Calculated as standardized mean differences (Cohen's d).

Table 3. Results analyzed by MCMC for generalized linear mixed model (GLMM).

The results of each outcome analysis by GLMM using MCMC were shown. There was a significant difference in the subjective QOL (Ikigai-9) between the experimental and control groups.

\begin{tabular}{lccc}
\hline & Time M (SD) & Group M (SD) & Group*Time M (SD) \\
\hline \multirow{2}{*}{ Ikigai-9 } & $-1.36(1.54)$ & $-2.36(5.16)$ & $4.44(2.17)$ \\
& $95 \%$ CI $[-4.49-1.62]$ & $95 \%$ CI $[-12.70-7.49]$ & $95 \%$ CI $[0.10-8.71]$ \\
\hline \multirow{2}{*}{ EQ-5D } & $0.110(0.063)$ & $-0.108(0.150)$ & $0.061(0.089)$ \\
& $95 \%$ CI $[-0.015-0.235]$ & $95 \%$ CI $[-0.405-0.188]$ & $95 \%$ CI $[-0.115-0.236]$ \\
\hline \multirow{2}{*}{ Flow state scale for } & $3.28(3.53)$ & $10.50(10.42)$ & $-0.73(5.01)$ \\
occupational task & $95 \%$ CI $[-3.65-10.33]$ & $95 \%$ CI $[-9.17-31.60]$ & $95 \%$ CI $[-10.88-8.94]$ \\
\hline \multirow{2}{*}{ FIM } & $18.99(3.45)$ & $-5.30(8.42)$ & $3.26(4.88)$ \\
& $95 \%$ CI $[11.97-25.57]$ & $95 \%$ CI $[-21.98-11.27]$ & $95 \%$ CI $[-6.37-12.72]$ \\
\hline
\end{tabular}

Note. $\mathrm{M}=$ mean; $\mathrm{SD}=$ standard deviation; $\mathrm{CI}=$ confidence interval; $\mathrm{EQ}-5 \mathrm{D}=$ EuroQol-5 Dimension;

FIM = Functional Independence Measure; Time = pre and post; Group = experimental and control groups. 

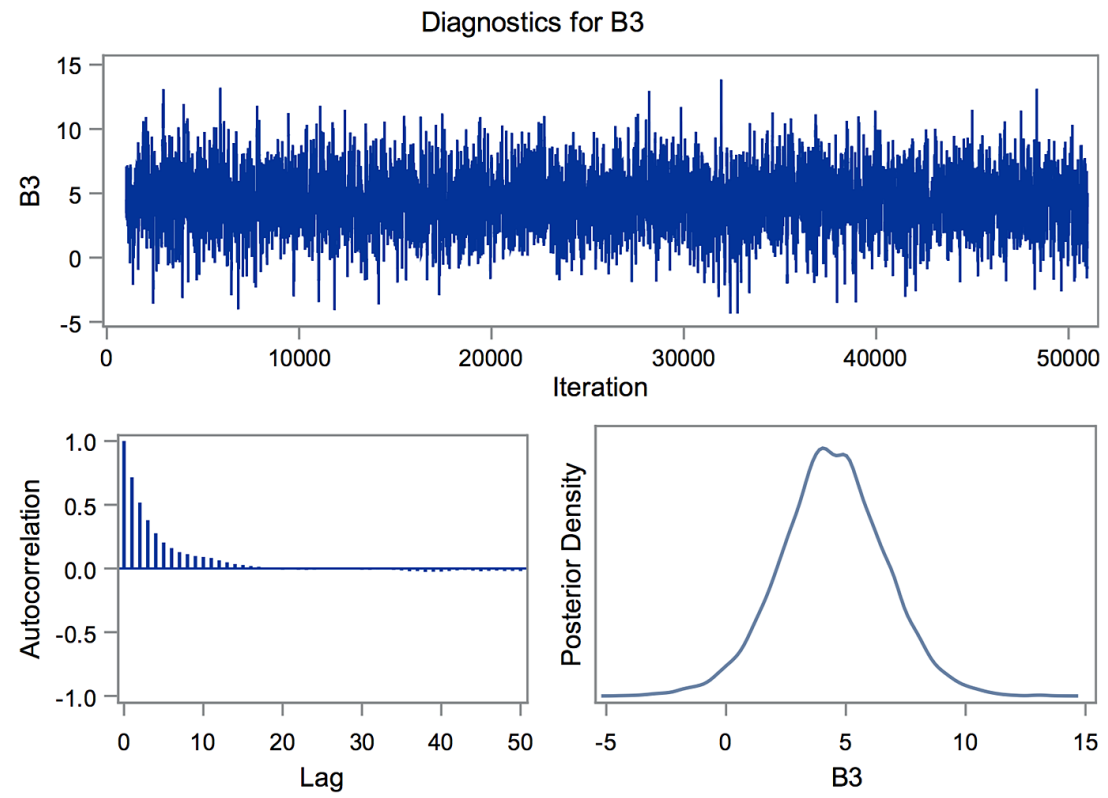

Note. B3 = Difference between experimental group and control group in Ikigai-9.

Fig. 2. PROC MCMC procedure about Ikigai-9.

This figure shows mixing of the Markov chain, which has relatively constant mean and variance over iterations. The trace plot shows indication of convergence.

pre-test and post-test results in both groups $(18.99 \pm$ $3.45,95 \%$ credible interval: $11.974-25.571)$.

Analysis by actual measurement values showed effect sizes (d) and $95 \%$ confidence intervals of 0.78 ( -0.09 to 1.65$), 0.19$ ( -0.70 to 0.97$)$, and 0.67 ( -0.21 to 1.50) for the Ikigai-9, EQ-5D, and Flow State Scale for Occupational Tasks, respectively. In addition, all of the clients in both groups were discharged home.

\section{Discussion}

To the best of our knowledge, no other study has compared the outcomes between ACS-OT and standard occupational therapy in a recovery rehabilitation unit. When we compared subjective QoL (Ikigai-9) between the two groups, there was a significant difference in the Bayesian GLMM. Although the effect size was moderate $(d=0.78)$, no significant effect was shown $(95 \%$ confidence interval, -0.09 to 1.65 ). In addition, there were no significant differences in health-related QoL (EQ-5D) or flow status (Flow State Scale for Occupational Tasks). Regarding FIM, there was no difference between the two groups, though both improved from before to after the intervention.

The ACS-OT method was based on the flow model, adjusting the client's therapy to their perceived skill with the support of an occupational therapist. The challengeskill balance was key to this flow model, requiring that the difficulty level of the occupational therapy engaged in by the client matched the ability of the client; it was therefore logical that occupational therapy and flow models should have a very high affinity. Although flow experiences can be addictive [35], this might relate to a lack of opportunity for meaningful chances to develop the skills needed to perform activities [36]. We believe that our ACS-OT method, by helping to improve skill by adjusting the challenge level to the patient's needs, helped promote the client's own initiative.

Bayesian estimation by MCMC was used to provide a statistical framework. In this method, data were combined with subjective prior information about parameter values to derive posterior probabilities of different models or parameter values. MCMC is a Bayesian statistical technique that samples parameters according to a stochastic algorithm that converges on the posterior probability distribution of parameters, combining information from the likelihood and the posterior distributions [37]. By using the prior distribution properly, this method can give a reasonable estimation even with small sample sizes. Adopting this analysis allowed us to calculate a more accurate estimated value with fewer samples.

The Ikigai-9 [28] used in this study consisted of the following three concepts: 1) optimistic and positive attitude toward present living and life, 2) active and positive attitude toward the future, and 3) recognition of the meaning of self-existence. In general, rehabilitation and occupational therapy focus on long-term 
goals. Pessimism of goal achievability might therefore be evidenced by high anxiety if the patient perceives a high challenge level and a low skill level. Equally, if the client judges that the goal has already been achieved (low challenge with high skill), he or she may be less likely to concentrate on an assigned activity. This relates to the psychological phenomenon of "mind wandering" [38], in which thoughts drift away from the task at hand and onto unrelated thoughts, such as memories, thoughts of the future, negative emotions, and misfortune [39]. Therefore, because the ACS-OT method focused on activities adjusted to achieve a challenge-skill balance in this study, it is possible that negative emotions and mind wandering may have been reduced, increasing being present or self-esteem. Since there was no significant difference between the two groups in the comparison on the contents of the selected activity, and tasks related to the activities were set taking into consideration the client's condition (skill), it is unlikely that the difference in the activities targeted for support affected the outcomes of the two groups. We believe that the use of the ACS-OT method has made it possible to support clients more appropriately and has led to positive psychological state.

We noted that the flow scale score did not differ between the two groups. Given that the ACS-OT method was based on the flow model, it is possible that clients in this study did not reach the flow state experienced with a high challenge-skill balance.

Regarding the FIM results, improvement was observed for ADL before and after intervention in both groups, but no significant difference was found between ACS-OT and normal occupational therapy. This suggested that for ADL, it could not be concluded that ACS-OT intervention was more effective than standard occupational therapy. The reason for this was that improvement of ADL was one of the main objectives in the recovery rehabilitation unit, and the improvement of ADL was also shown in conventional occupational therapy [40-42], it was considered that clear difference was not reached even with ACS-OT.

A limitation of this research is that it was only a controlled clinical trial. Therefore, we are planning a randomized controlled trial to obtain more evidence in the future (UMIN ID: UMIN000029505). Moreover, we used the GLMM to estimate values in this study because of the small number of subjects. Although we confirmed the sampling accuracy, we would like to clarify the effects of the ACS-OT method, including verification of the validity of the Bayesian estimate, in a future randomized controlled trial.

\section{Conclusion}

In conclusion, this study showed that the ACS-OT method could improve a client's subjective QoL (i.e., ikigai) during recovery in a rehabilitation unit. Combined with the results of previous studies [22, 23], this method could have application in different areas of occupational therapy.

Acknowledgements: This research was carried out under the assignment research grant system by the Japan Occupational Therapist Association in 2017.

\section{References}

[1] World Federation of Occupational Therapists [online]. Statement on Occupational Therapy [cited 2012]. Available from: http://www.wfot.org/ResourceCentre.aspx

[2] American Occupational Therapy Association. Occupational therapy practice framework: Domain and process (3rd ed.). Am J Occup Ther. 2014; 68: S1-S48.

[3] Fisher AG, and Jones KB. Occupational therapy intervention process model: a model for planning and implementing top-down, client-centered and occupation-based interventions. Three Star Press; 2009.

[4] Nishii M. Management Tool for Daily Life Performance (in Japanese). Journal of Allied Health Science. 2016; 7(1): 17-21.

[5] Boniface G, Fedden T, Hurst H, Mason M, Phelps C, Reagon $\mathrm{C}$, et al. Using theory to underpin an integrated occupational therapy service through the Canadian Model of Occupational Performance. Br J Occup Ther. 2008; 71(12): 531-9.

[6] Kielhofner G, editors. Model of human occupation: Theory and application. Lippincott Williams \& Wilkins; 2008.

[7] Maitra KK, and Erway F. Perception of client-centered practice in occupational therapists and their clients. Am J Occup Ther. 2006; 60(3): 298-310.

[8] Csikszentmihalyi M. Play and intrinsic rewards. J Humanist Psychol. 1975.

[9] Csikszentmihalyi M. Flow: The Psychology of Optimal Experience, New York. Harper and Row; 1990.

[10] Csikszentmihalyi M, Csikszentmihalyi IS, editors. Optimal experience: Psychological studies of flow in consciousness. Cambridge university press; 1992.

[11] Haworth J, and Evans S. Challenge, skill and positive subjective states in the daily life of a sample of YTS students. J Occup Organ Psychol. 1995; 68(2): 109-21.

[12] Nielsen K, and Cleal B. Predicting flow at work: Investigating the activities and job characteristics that predict flow states at work. J Occup Health Psychol. 2010; 15(2): 180-90.

[13] Ilies R, Wagner D, Wilson K, Ceja L, Johnson M, DeRue $\mathrm{S}$, et al. Flow at Work and Basic Psychological Needs: Effects on Well-Being. Applied Psychology. 2017; 66(1): 
3-24.

[14] Rebeiro KL, and Polgar JM. Enabling occupational performance: optimal experiences in therapy. Can J Occup Ther. 1999; 66(1): 14-22.

[15] Wright JJ, Sadlo G, and Stew G. Further explorations into the conundrum of flow process. J Occup Sci. 2007; 14(3): $136-44$.

[16] Larson E, and von Eye A. Beyond flow: temporality and participation in everyday activities. Am J Occup Ther. 2010; 64(1): 152-63.

[17] Jonsson H, and Persson D. Towards an experiential model of occupational balance: An alternative perspective on flow theory analysis. J Occup Sci. 2006; 13(1): 62-73.

[18] Engeser S, and Rheinberg F. Flow, moderators of challenge-skill-balance and performance. Motiv Emot. 2008; 32(3): $158-72$.

[19] Jacobs K. Flow and the occupational therapy practitioner. Am J Occup Ther. 1994; 48(11): 989-96.

[20] Hirao K, Kobayashi R, Okishima K, and Tomokuni Y. Flow experience and health-related quality of life in community dwelling elderly Japanese. Nursing \& health sciences. 2012; 14(1): 52-7.

[21] Yasunaga M, Kobayashi N, and Yamada T. The challenge skill ratio of users' experiences at an adult daycare center: Emotional states based on the Flow model (in Japanese). The Journal of Japanese Occupational Therapy Association. 2012; 31(1): 83-93.

[22] Yoshida I, Mima H, Nonaka T, Kobayashi R, and Hirao $\mathrm{K}$. Analysis of subjective evaluation in occupations of the elderly: A study based on the flow model (in Japanese). The Journal of Japanese Occupational Therapy Association. 2016; 35(2): 70-9.

[23] Yoshida I, Hirao K, and Nonaka T. Adjusting ChallengeSkill Balance to Improve Quality of Life in Older Adults: A Randomized Controlled Trial. Am J Occup Ther. 2018; 72(1): $1-8$.

[24] Anthony JC, LeResche L, Niaz U, Von Korff MR, and Folstein MF. Limits of the 'Mini-Mental State'as a screening test for dementia and delirium among hospital patients. Psychological medicine. 1982; 12(2): 397-408.

[25] Graff MJ, Vernooij-Dassen MJ, Thijssen M, Dekker J, Hoefnagels WH, and OldeRikkert MG. Effects of community occupational therapy on quality of life, mood, and health status in dementia patients and their caregivers: a randomized controlled trial. J Gerontol A Biol Sci Med Sci. 2007; 62(9): 1002-9.

[26] Law M, Baptiste S, and Mills J. Client-centered practice: What does it mean and does it make a difference? Can J Occup Ther. 1995; 62(5): 250-7.

[27] Csikszentmihalyi M, and Larson R. Being adolescent: Conflict and growth in the teenage years. Basic Books; 1986.

[28] Imai T, Osada H, and Nishimura Y. The reliability and validity of a new scale for measuring the concept of Ikigai (Ikigai-9) (in Japanese). Japanese Journal of Public Health. 2012; 59(7): 433-9.

[29] EuroQol G. EuroQol-a new facility for the measurement of health-related quality of life. Health policy. 1990; 16(3): 199-208.

[30] Yoshida K, Asakawa K, Yamauchi T, Sakuraba S, Sawamura D, Murakami Y, et al. The flow state scale for occupational tasks: development, reliability, and validity. Hong Kong J Occup Ther. 2013; 23(2): 54-61.

[31] Granger CV, Hamilton BB, Keith RA, Zielezny M, and Sherwin FS. Advances in functional assessment for medical rehabilitation. Topics in geriatric rehabilitation. 1986; 1(3): 59-74.

[32] Kanda Y. Investigation of the freely available easy-touse software 'EZR' for medical statistics. Bone marrow transplantation. 2013; 48(3): 452-8.

[33] Brémaud P. Markov chains: Gibbs fields, Monte Carlo simulation, and queues. Springer Science \& Business Media; 2013.

[34] Raftery AE, and Lewis S. How many iterations in the Gibbs sampler? Washington Univ Seattle Dept of Statistics; 1991.

[35] Csikszentmihalyi M. Activity and happiness: Towards a science of occupation. J Occup Sci. 1993; 1: 38-42.

[36] Helbig K, and McKay E. An exploration of addictive behaviours from an occupational perspective. J Occup Sci. 2003; 10(3): 140-5.

[37] Bolker BM, Brooks ME, Clark CJ, Geange SW, Poulsen JR, Stevens $\mathrm{MHH}$, et al. Generalized linear mixed models: a practical guide for ecology and evolution. Trends in ecology \& evolution. 2009; 24(3): 127-35.

[38] Smallwood J, Fitzgerald A, Miles LK, and Phillips LH. Shifting moods, wandering minds: negative moods lead the mind to wander. Emotion. 2009; 9(2): 271.

[39] Killingsworth MA, and Gilbert DT. A wandering mind is an unhappy mind. Science. 2009; 330(6006): 932.

[40] Jackson J, Carlson M, Mandel D, Zemke R, and Clark F. Occupation in lifestyle redesign: The well elderly study occupational therapy program. Am J Occup Ther. 1998; 52(5): 326-36.

[41] Noto S, Murai C, Takeuchi S, and Nakamura H. Effect of occupational therapy using "the management tool for daily life performance" for dependent community-dwelling elderly: A randomized controlled multi-center trial (in Japanese). The Journal of Japanese Occupational Therapy Association. 2014; 33(3): 259-69.

[42] Tomori K, Nagayama H, Ohno K, Nagatani R, Saito Y, Takahashi $\mathrm{K}$, et al. Comparison of occupation-based and impairment-based occupational therapy for subacute stroke: A randomized controlled feasibility study. Clinical rehabilitation. 2015; 29(8): 752-62. 